\section{WEB WATCH}

Celebrating UK geneticists

- http://www.dna50.org.uk

In celebration of the 50th

anniversary of the

publication of the structure

of DNA, the Medical

Research Council, Nature

and the Royal Society have

worked together to produce

this highly informative web

site. It describes past

achievements and future

prospects for genetic

research in the UK. It also

advertises the scientific

events that are taking place

this year as part of the 50

year celebrations. Ethical and

social issues that are

associated with genetic

research are addressed in the

Science in Society section.

The key events, from

Mendel's studies in the

1850s establishing the

particulate nature of

inheritance to the

elucidation of the structure of DNA in 1953, are

included in a useful timeline. This timeline focuses on the principal contributions that

UK researchers have made to genetics since 1953 ,

including the invention of

Fred Sanger's sequencing technology in 1977 and the development of DNA fingerprinting methods by Alex Jeffreys in the 1980s. Looking to the future, the web site reviews the

potential applications that could be developed from our existing knowledge of genetics and molecular biology. The source of much of this information is the UK Foreign and

Commonwealth Office publication entitled 'DNA and after, 50 years of excellence'.

The site is easy to navigate, contains useful scientific information and provides a calendar of the events that will be happening throughout the year in the UK - including hands-on workshops for students, and public debates on topical issues. There is also a handy Science in the News section that is contributed by the Royal Society.

Catherine Baxter

\title{
Another brick in the wall
}

The host of celebratory scientific events that are scheduled for 2003 illustrates just how many bricks have been added to Watson and Crick's
1953 foundation stone over the years. James Watson was on hand to be honoured at the first of these events "50 Years On: From the Double
Helix to Molecular Medicine" (1-5 February, Miami, USA). In the same month, Watson also attended the premier celebratory US meeting

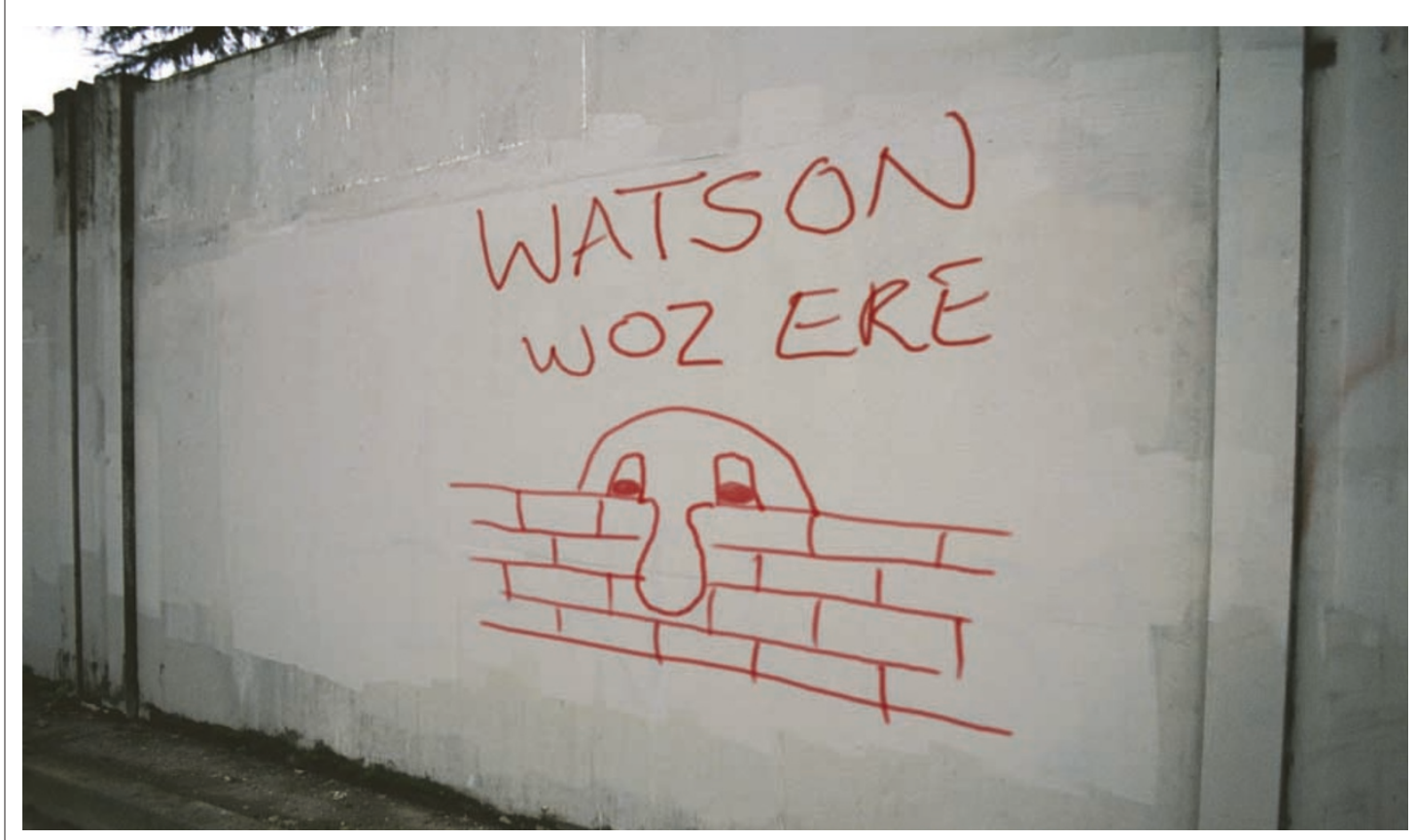

5 OTH ANNIVERSARY

\section{After the double helix}

The 50th anniversary celebrations marking the discovery of the threedimensional structure of DNA provide an opportunity to reflect on the key developments in the field of genetics over the past 50 years, and to consider the future of genetic research.

Before Watson and Crick's 1953 paper, Avery, MacLeod and McCarty in 1944, and Hershey and Chase in 1952, had provided the experimental data that established DNA as the heritable genetic material. With the knowledge of the structure of DNA, research then focused on its replication and information-encoding properties. In 1958, Meselson and Stahl showed that DNA replication is semi-conservative: new molecules consist of one original strand from the parental molecule and one new strand. Contributions by Crick, Brenner, Nirenberg, Khorana, Matthaei and Holley, among others, enabled the genetic code to be cracked by 1966 . However, a remaining challenge is to understand the information that is encoded in regulatory DNA.

DNA sequencing has revolutionized genetics. The Sanger and Maxam-Gilbert methods were published in 1977. The first genome to be sequenced was the bacteriophage FX174 $(\sim 5 \mathrm{~kb})$ in 1980 and the first freeliving organism to be sequenced was Haemophilus influenzae $(\sim 1.8 \mathrm{Mb})$ in 1995. Recent advances have reduced the cost and enhanced the speed of sequencing, so the sequences of several whole genomes, from a wide taxonomic distribution, have now been published - including the much anticipated human genome in 2001. Another important technical breakthrough occurred in 1983 when Mullis developed the polymerase chain reaction (PCR). Many other tools for molecular biology have been developed over the past 50 years, including restriction enzymes, nucleic acid hybridization techniques, cloning and genetic engineering. The application of these methods has led to some interesting applications. Notably, gene therapy was first used in 1990 to treat a patient suffering with the immune disorder adenosine deaminase (ADA) deficiency; the first transgenic food - the 
H I G H L I GHTS

("The Biology of DNA", 26 February-2 March, Cold Spring Harbor Laboratory).

The European scientific celebrations will be launched with "Nobel Day" at the World Life Sciences Forum (8 April, Lyons, France), which boasts no fewer than 11 Nobel prize winners, including Watson, and covers the entire breadth of the discovery's impact. Watson also finds time to attend a genome-focused symposium ("From Double Helix to Human Sequence - and Beyond" 14-15 April, Bethesda, USA), a Royal Society discussion meeting ("Replicating and Reshaping DNA", 23-24 April, The Royal Society, London, UK) and a conference held on the exact anniversary of the famous publication ("DNA: 50 years of the Double Helix", 25 April, Cambridge, UK).

Following the frenzy of doublehelix related activity in April, the programme of scientific events slows down, but continues for the rest of the year. The annual Cold Spring Harbor Symposium this year celebrates both the anniversary of the double helix and the impending completion of the human genome ("The Genome of Homo sapiens", 27 May-3 June). The International
Congress of Genetics — a flagship event for the community that is only held once every five years - also has a genomic flavour ("Genomes The Linkage to Life", 6-12 July, Melbourne, Australia).

Meetings in many other disciplines on which genetics has had an impact on in the past 50 years will be hosting symposia or discussions for the anniversary; for example, the symposium on "Exploiting Genomes: Bases to Megabases in 50 years" at the Society of General Microbiology's meeting (8-9 September, Manchester, UK).

Biotechnology is another area that owes a debt to the double helix, and later in the year a symposium at UC Berkeley (10-11 October, San Francisco, USA) will explore its impact over the past 50 years (the ubiquitous Watson will be in attendance!).

So, it will be a busy year for geneticists world-wide, but, as I'm sure most attendees at these meetings will agree, while it must have been great for Watson and Crick to lay the foundation stone, it is also good to be a humble bricklayer on a construction as exciting as this one.

Nick Campbell

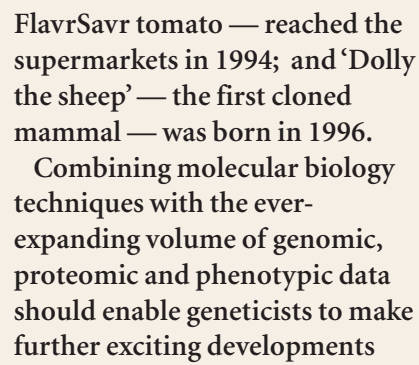

over the next 50 years. There is little doubt that genetics will continue to benefit society, in particular through improvements in healthcare and agriculture. Catherine Baxter

\section{(19) References and links} FURTHER READING The double helix -50 years. Nature 491 , 396-453 (2003)

\section{Timeline | DNA milestones}

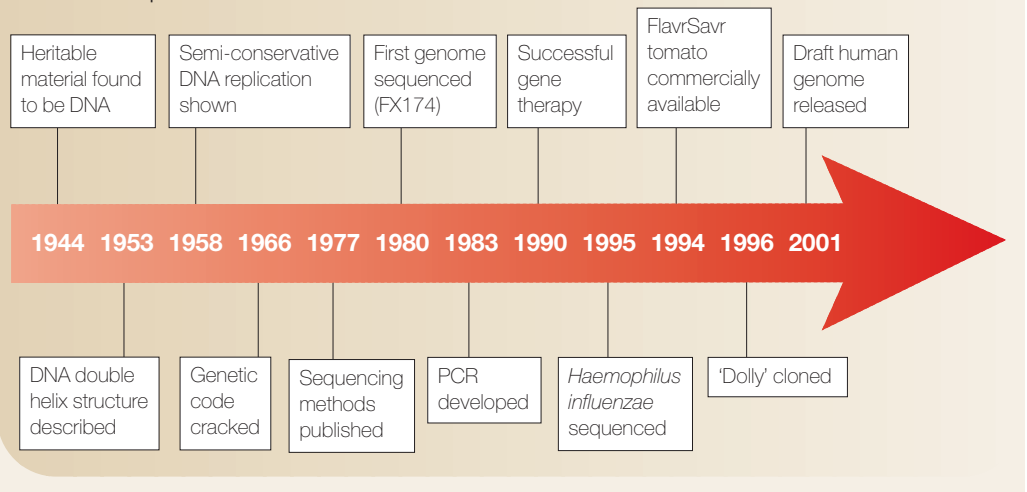

\section{IN BRIEF}

\section{GM ORGANISMS}

Impact of genetic manipulation on the fitness of Anopheles stephensi mosquitoes.

Catteruccia, F. et al. Science 299, 1225-1227 (2003)

It has been argued that malaria could be controlled by introducing into natural populations transgenic mosquitoes that express genes that impair parasite transmission. For this strategy to be successful, the transgenic mosquitoes must be able to survive and reproduce competitively in the wild. However, this study shows that transgene expression, mutations introduced by transgene insertion, and inbreeding can result in a lower fitness of transgenic mosquitoes relative to wild type.

\section{MOUSE MODELS}

Modification of ocular defects in mouse developmental glaucoma models by tyrosinase.

Libby, R. T. et al. Science 299, 1578-1581 (2003)

Human primary congenital glaucoma (PCG) is often caused by mutations in the cytochrome P450 family member CYP1B1, and is associated with abnormal ocular drainage structures. This paper shows that Cyp1b1-deficient mice provide a good model for this type of glaucoma. Libby et al. used these knockout mice to show that tyrosinase gene deficiency increases the severity of the disease phenotype and that this is alleviated by applying dihydoxyphenylalanine (L-dopa). This raises the possibility of new glaucoma therapies.

\section{TECHNOLOGY}

\section{RNA interference targeting Fas protects mice from} fulminant hepatitis.

Song, E. et al. Nature Med. 19, 347-351 (2003)

RNAi can target and silence mammalian genes but can it prevent disease? Song et al. show that, in mice, RNAi can silence the gene Fas that codes for an important mediator of hepatocyte apoptosis. This indicates that RNAi could be used to prevent the adverse effects of hepatitis that are linked to cell death. The authors test this hypothesis in two models of Fas-mediated liver damage, and show for the first time that siRNA can prevent disease in vivo.

\section{FUNCTIONAL GENOMICS}

\section{Scanning the human genome with combinatorial transcription factor libraries.}

\section{Blancafort, P. et al. Nature Biotech. 21, 269-274 (2003)}

Blancafort et al. report a new technology that could be useful for studying and modulating gene function. They have constructed large libraries of artificial transcription factors that contain between three and six zinc-finger domains $\left(\mathrm{TF}_{\mathrm{ZF}} \mathrm{s}\right)$ that can either activate or repress gene expression. $\mathrm{TF}_{\mathrm{ZF}} \mathrm{s}$ can be applied to a cell line that is then screened for a desired phenotype. In this example, $\mathrm{TF}_{\mathrm{ZF}} \mathrm{s}$ were identified that were able to induce expression of the endothelial marker VE-cadherin in non-endothelial cell lines and to repress its expression when combined with a repression domain. 